\title{
The Knowledge, Attitude and Practices of Mothers of Hospitalized Children 6 Month To 5 Years of Age Towards Acute Diarrhoea and Oral Rehydration Therapy
}

\author{
Authors \\ Dr Dipendra Sharma ${ }^{1}$, Dr Himanshu Goyal ${ }^{2}$, Dr Nishant Aswani ${ }^{3}$, Dr R .K. Gulati ${ }^{4}$ \\ ${ }^{1}$ Senior Medical Officer Cum Clinical Tutor, Dept of Paediatrics, JK Lone Hospital, GMC Kota \\ ${ }^{2}$ Resident Doctor, Dept of Paediatrics, JK Lone Hospital, GMC Kota \\ ${ }^{3}$ (MD Pediatrics) Dept of Paediatrics, JK Lone Hospital, GMC Kota \\ ${ }^{4}$ Sr Professor and Head, Dept of Paediatrics, JK Lone Hospital, GMC Kota
}

\begin{abstract}
Diarrhoea is one of the commonest cause of morbidity among young children .Diarrhoeal infections are fifth leading cause of mortality worldwide.Global reports indicates that in 2008, despite the recommendations about the success of ORS, only 38\% of children less than 5 year old received oral rehydration therapy (ORT) and continued food intake during diarrhoeal episodes. This study aimed to assess the knowledge, attitude and practices of mothers of hospitalized children 6 month to 5 years of age towards acute diarrhoea and oral rehydration therapy

In this prospective study mothers of children aged 6 month to 5 years having acute diarrhoea admitted in pediatric ward of tertiary care J K Lone hospital Kota from november 2013 to october 2014 were included. After taking written and informed consent, the mothers were interviewed and accordingly history and brief clinical examination was done.

The number of patients decreased with increasing education status of mother. There were 42.9\%mothers (illiterate), below graduate were $56.88 \%$ and graduate mothers were $0.8 \%$ in this study. In our study only a meagre of $17.9 \%$ mothers knew how to prepare oral rehydration solution. In our study a total of 52\% mothers washed hands before food. In our study 99 of 228 (43.4\%) mothers knew that ongoing diarrhoea breeds malnutrition.
\end{abstract}

Promotional training about the ORS and nutrition and hygiene can prevent and manage diarrhoeal illness.

Keywords: oral rehydration solution, diarrhoea, malnutrition.

\section{Introduction}

Diarrhoea is one of the commonest cause of morbidity among young children in developing countries as well as low income countries. Young children are most vulnerable especially under 5

year of age group. It is associated with lack of safe drinking water, inadequate sanitation and poor hygiene. Diarrhoeal infections are fifth leading cause of mortality worldwide ${ }^{(1,2)}$ 
Fluid therapy with ORS and other home solutions and continued breast feeding are the typical treatment. Since 1970s ORS has been the cornerstone of management in order to prevent life threatening dehydration associated with diarrhoea. Nevertheless, only less than $40 \%$ of children with diarrhoea in developing countries received the recommended treatment and there has been a progress towards the trend in the last decades ${ }^{(3,4)}$

Global reports indicates that in 2008, despite the recommendations about the success of ORS, only $38 \%$ of children less than 5 year old received oral rehydration therapy (ORT) and continued food intake during diarrhoeal episodes.

This study aimed to assess the knowledge ,attitude and practices of mothers of hospitalized children 6 month to 5 years of age towards acute diarrhoea and oral rehydration therapy.

\section{Material and method}

In this prospective study mothers of children aged 6 month to 5 years having acute diarrhoea and admitted in pediatric ward of tertiary care $\mathrm{J} \mathrm{K}$ Lone hospital Kota, from november 2013 to october 2014 were included. Children having acute dysentry and having diarrhoea for more than 14 days were excluded. After taking written and informed consent from the guardian of children, the mothers were interviewed and accordingly history and brief clinical examination was done. By this knowledge, attitude and practices of mothers towards acute diarrhoea and oral rehydration therapy was assessed.

\section{Results}

A total of 228 patients of age range 6 month to 60 months were enrolled in this study. Out of affected 228 patients, 117 (51.3\%) were male and $111(48.6 \%)$ were female.

There were 15 children in the severe dehydration. Out of 15 children, the mothers of only 3 (20\%) received primary education; rest all were illiterate.
None of these mothers knew about danger signs of diarrhoea. Although 6 (40\%) knew how to prepare ORS but none of the mothers gave ORS to the child before bringing to the doctor. Only 6 (40\%) mothers knew correctly about the administration of ORS. Washing of hands before food was pracised by only $3(20 \%)$. Only 6 (40\%) mothers knew that diarrhoea could be prevented by hand washing and maintaining proper hygiene.

There were 102 children in some dehydration. Maternal literacy was seen in $44.1 \%$ of children suffering from some dehydration. Only 18 (17.6\%) mothers were aware of general danger signs. Only 17 (16.6\%) knew how to prepare ORS and purpose to give ORS to replenish fluid and electrolytes was known to $8(7.8 \%)$. Out of 102 children with severe dehydration 39 mothers (38.2\%) didn't give any ORT to their children. Washing of hands before food was practiced by only $64.7 \%$ mothers.

There were 111 children with no sign of dehydration. Maternal literacy was $36.93 \%$ in this group. Out of 111 ,only 18 (16.2\%) knew how to prepare ORS and 30 (27\%) knew about danger signs of dehydration $.72 .9 \%$ mothers knew to administer ORS after each episode of diarrhoea. 33 mothers $(29.8 \%)$ didn't give any ORT to their children. $78(70.2 \%)$ knew about the alternatives to ORS. 66 mothers (59.4\%) didn't wash hands before food in this group.

Ongoing diarrhoea breeds malnutrition. In our study 99 of 228 (43.4\%) mothers knew this fact. 3 of 15 mothers in severe dehydration group,12 of 102 in some dehydration group and 84 of 111 in no dehydration group knew that malnutrition complicates diarrhoeal illness. 
Table :Impact of various factors in children with severe, some and no dehydration

\begin{tabular}{|l|l|l|l|l|}
\hline S.no. & Factors & $\begin{array}{l}\text { Severe } \\
\text { dehydration }\end{array}$ & $\begin{array}{l}\text { Some } \\
\text { dehydration }\end{array}$ & No dehydration \\
\hline 1. & No. Of children & 15 & 102 & 111 \\
\hline 2. & Maternal literacy & 3 & 45 & 41 \\
\hline 3. & Knowledge about ORS & 6 & 17 & 18 \\
\hline 4. & Hand washing & 3 & 66 & 81 \\
\hline 5. & Knowledge about malnutrition & 3 & 12 & 84 \\
\hline
\end{tabular}

\section{Discussion}

Knowledge and practices of mothers or other care takers of children are important determinants of the occurrence or outcome of diarrhoeal diseases. Base line information of these variables is also needed for developing health education programmes and for formulating national policy on home fluid therapy.

As regards maternal education and knowledge is concerned, the number of patients decreased with increasing education status of mother. There were 98 mothers (42.9\%) illiterate, below graduate were $56.88 \%$ and graduate mothers were $2(0.8 \%)$ in this study. The relationship between maternal education and state of health of the children is well documented $^{(5)}$. In a survey by Ali $\mathrm{M}$ et al maternal literacy was found significantly associated with the knowledge regarding various aspects of ORT. Maternal literacy persistently affects mother's understanding of ORT and her ability to prepare ORS. Poor literacy status appears to limit the mother's ability to utilize ORS for preventing diarrhoea induced dehydration. ${ }^{(5)}$

In our study only a meagre of $17.9 \%$ mothers (2.6\% in severely dehydrated children, $7.4 \%$ in some dehydration group and $7.8 \%$ in no dehydration group) knew how to prepare oral rehydration solution. As it can be seen from the data that lack of knowledge leads to worsened child's dehydration.

In our study a total of $52 \%$ mothers washed hands before food. Washing of hands before food was practised by $20 \%$ mothers in severe dehydration group, $64.7 \%$ in some dehydration group while the figure was $73 \%$ for mothers of children with no dehydration. It clearly indicates the role of hand washing in prevention of diarrhoeal illness.

\section{Conclusion}

Continuous training in society to promote the use of more fluids, continuation of breast feeding, adequate and appropriate nutrition and taking care of children during episodes of diarrhoea are the main factors that bring children back to health. Promotion of hygiene behaviour is effective if it is done repeatedly over longer periods of time. It should be noted that maternal knowledge and performance is also influenced by the behaviour of doctors and health care staff in health centres. Therefore, training programmes are recommended to be held for medical students, practitioners and nursing staff to increase the knowledge and practices about the diarrhoea and use of ORS.

\section{Conflicts of interest}

There are no conflicts of interest.

\section{Bibliography}

1. Kolahi AA, Rastegarpour A, Sohrabi MR ;the impact of an urban sewage system on childhood diarrhoea in Tehran, Iran;a concurrent control field trial.Trans R Soc Trop Med Hyg 2009 ;103(5):500-51 and life sciuence 2010;6(2):92-98

2. Walker SP, Waches TD, Gardner JM, Lozoff B ,Wasserman GA,Pollitt E et al:child development :risk factors for adverse outcomes in developing countries .Lancet 2007:369 (9556):145-57

3. Pahwa S , Kumar GT, Toteja GS:performance of a community based 
health and nutrition educated intervention in the management of diarrhoea in a slum of Dehli ,India,J health Popul nutr.2010;28(6):553-9.

4. Alkizim F, Matheka D, Murithi A , childhood diarrhoea :failing conventional measures, what next ? Pan Afr Med J $2011 ; 8 ; 47$

5. Ali M ,Underwood, Determinants of use rate of oral rehydration therapy for management of childhood diarrhoea in rural Bangladesh. Journal of health and population nutrition.2000;18(2):103-108. 